\title{
Chitosan Associated with the Extract of Unripe Banana Peel for Potential Wound Dressing Application
}

\author{
Patrícia Bataglini Franco, ${ }^{1}$ Leiliane Aparecida de Almeida, ${ }^{1}$ \\ Rodrigo Fernando Costa Marques, ${ }^{2}$ Mariana Altenhofen da Silva, ${ }^{3}$ \\ and Maria Gabriela Nogueira Campos ${ }^{1}$ \\ ${ }^{1}$ Institute of Science and Technology, Federal University of Alfenas, Rod. José A. Vilela 11.999, 37715-400 Poços de Caldas, MG, Brazil \\ ${ }^{2}$ Institute of Chemistry, São Paulo State University "Júlio de Mesquita Filho", Rua Prof. Francisco Degni 55, \\ 14800-060 Araraquara, SP, Brazil \\ ${ }^{3}$ Center of Agricultural Sciences, Federal University of São Carlos, Rod. Anhanguera Km 174, Araras, SP, Brazil
}

Correspondence should be addressed to Patrícia Bataglini Franco; patbattaglini@hotmail.com

Received 19 April 2017; Accepted 29 May 2017; Published 3 July 2017

Academic Editor: Hossein Roghani-Mamaqani

Copyright (c) 2017 Patrícia Bataglini Franco et al. This is an open access article distributed under the Creative Commons Attribution License, which permits unrestricted use, distribution, and reproduction in any medium, provided the original work is properly cited.

\begin{abstract}
The authors report the preparation of chitosan membranes associated with the extract of unripe banana peel. Extracts were prepared by decoction, using boiling distilled water. The extraction process was repeated three times. First and third extracts served as solvent to prepare chitosan membranes by solvent evaporation technique. The chitosan membranes associated with the first and third extracts of unripe banana peel exhibit good flexibility, transparency, and uniformity. Scanning Electron Microscopy images showed dense membranes. Brownish color of membranes was observed due to the presence of tannins, which was confirmed by Infrared Spectroscopy analysis. Thermal properties of the membranes were evaluated by Differential Scanning Calorimetry (DSC), which exhibited good thermal stability at physiological temperature $\left(37^{\circ} \mathrm{C}\right)$. At this temperature and $\mathrm{pH} 7.2$, membranes were also able to absorb fluids. The hydrophilic character of the membranes was determined by contact angle measurements. The membranes demonstrated their capacity for gaseous exchange and exhibited water-vapor permeability (WVP) rates comparable to injured skin one. Finally, high number of viable dermal fibroblasts was observed by indirect cytotoxicity assay suggesting potential application of these membranes as skin wound dressing.
\end{abstract}

\section{Introduction}

Trauma, vascular insufficiency, and diseases, such as diabetes, hypertension, rheumatism, and inflammatory disease, may cause wounds [1-3]. They are generally classified according to the thickness in which the subcutaneous layer is compromised as acute or chronic wounds [4]. Acute wounds are superficial while chronic ones can result by destructing all the layers in skin [5]. The tissue damage stimulates a coordinated physiological response to provide hemostasis and initiate the processes of inflammation, proliferation, and remodeling $[1,5]$. This healing process is well known and occurs within 3 weeks for acute wounds but usually persist for a minimum of 12 weeks since the time of injury for chronic wounds $[5,6]$. The delayed healing or nonhealing of chronic wounds is due to the prolonged pathological inflammation and/or disruption of the healing process $[1,5]$. Moreover, they can heal without restoring normal anatomy and function of the damage tissue [1]. Chronic wounds are considered a public health problem and lead to serious morbidity and mortality [4]. They have been considerably increasing since one-third of the adult population is currently living with diabetes and it is estimated that between 10 and $25 \%$ of patients with diabetes will develop a foot ulcer in their lifetime [7]. Wound treatments are costly and involve several medical areas. The estimated annual expenditure on wound related treatments in the USA is over one billion dollars [5]. The current standard of care for chronic wounds consists of swabbing for infection, cleaning, and dressing [4]. The choice of dressing depends on several factors, such as the cause, size, depth, location, and 
degree of exudation and level of contamination of the wound [6]. An ideal dressing should protect the wound from further trauma and against contamination, ensure a moist environment by absorbing or remove excess exudate, and be flexible, biodegradable, and cost-effective $[6,8]$. Currently, there are hundreds of products, devices, and dressing materials on the market to aid in wound management. Nevertheless, the most currently available wound dressings are made using chitosan, hyaluronic acid, collagen, and silicon [4].

Chitosan based dressings have mostly been investigated to due to chitosan's favorable properties suitable for wound healing, such as intrinsic antimicrobial properties and hemostatic activity, besides being biodegradable, biocompatible, bioadherent, and nontoxic $[4,8,9]$. Chitosan is derived from chitin, which can be found as the primary structural polymer in the exoskeleton of crustacean [9]. As a byproduct of fishing industry, it is generating large notice as biomaterial because of not only its interesting biological properties, but also its low-cost when compared to collagen and hyaluronic acid $[4,9]$.

Banana peel is another low-cost byproduct of food industry that exhibits attractive biological properties for wound healing management [9]. It is antioxidant and antimicrobial (bacterial, fungal, and yeast activities) and demonstrates higher concentration of phenolic compounds and mineral content [9-11]. The healing and antimicrobial properties of banana peel are associated with the presence of tannin, which are found in higher concentration in the unripe banana peel $[12,13]$. Thus, association of chitosan and unripe banana peel might be a great alternative of a low-cost biomaterial for wound dressing application.

We recently demonstrated the antibacterial activity of chitosan membranes associated with unripe banana peel against Staphylococcus aureus and Escherichia coli [9]. Kapadia et al. (2015) reported antimicrobial activity of banana peel on Porphyromonas gingivalis and Aggregatibacter actinomycetemcomitans [14]. Kamel et al. (2016) also studied a chitosan/banana peel powder nanocomposite for wound healing application [11].

In this present work, we investigated morphological, thermal, chemical, hydrophilicity, and barrier properties of chitosan membranes associated with the extract of unripe banana peel by Scanning Electron Microscopy (SEM), Differential Scanning Calorimetry (DSC), Infrared Spectroscopy (FTIR), contact angle, and water-vapor permeability (WVP) techniques. Moreover, in vitro cytotoxicity was evaluated using dermal fibroblast cell line.

\section{Materials and Methods}

2.1. Materials. High molecular weight chitosan, deacetylation degree greater than 75\%, was purchased from SigmaAldrich (USA). Glacial acetic acid was purchased from Synth (Brazil). Bananas (Musa cavendishii), classified as category 1 in the maturity scale of Von Loeseck (1950) [15], were used for obtaining the peels. Commercial sodium hypochlorite $5 \%$ was purchased from Audax (Brazil).

\subsection{Methods}

2.2.1. Preparation of the Extract of Unripe Banana Peel. Extraction of the active compounds of the unripe banana peels was performed by the decoction method, in order to extract the water-soluble compounds. Firstly, the fruits were disinfected by immersion in a sodium hypochlorite solution $(1 \% \mathrm{v} / \mathrm{v})$ for 15 minutes. Then, fruits were washed with water and dried with absorbent paper. Pulps were discarded and the peels were cut and weighted. The disinfected peels $(250 \mathrm{~g})$ were added to $1 \mathrm{~L}$ of boiling distilled water for two hours, using a heating pad. The product was stored under refrigeration for 24 hours and then filtered to obtain the first extract (liquid portion) that was reserved for membrane preparation purpose and the extracted peels (solid portion), which was reused. In order to maximize the extraction of metabolites of the peels, the decoction process was repeated twice using the same extracted peels and the third extract (liquid portion of the third filtration) was also used to prepare the membranes.

2.2.2. Preparation of Membranes. The membranes were obtained by solvent evaporation technique. Three solutions were prepared containing $2 \%(\mathrm{w} / \mathrm{v})$ of chitosan dissolved in three different solvents: distilled water (CB0), first extract (CB1), and third extract (CB3). The $\mathrm{pH}$ of all solvents was previously adjusted to 6.0 using acetic acid $1 \mathrm{M}$ in order to promote the complete dissolution of chitosan. The solutions were then cast in plastic molds of $14 \mathrm{~cm}$ diameter and dried in an oven at $60^{\circ} \mathrm{C}$ for 4 hours.

2.2.3. Morphological Analysis. The membranes morphology was evaluated by Scanning Electron Microscopy (SEM). The obtained membranes were previously dried in a desiccator with anhydrous calcium chloride for a week. Samples were coated with a $16 \mathrm{nn}$ thickness gold in a Sputter Coater (BalTec, model SCD050) for 60 seconds at $2 \times 10^{-2} \mathrm{~Pa}$ and $24^{\circ} \mathrm{C}$. Morphological analysis was performed using JEOL JSM-7500 F Field Emission Scanning Electron Microscope.

2.2.4. Infrared Spectroscopy (FTIR). The FTIR technique was used to characterize the functional groups present in the CB1, CB3, and unripe banana peel samples. FTIR spectra were recorded on Thermo Scientific Nicolet iS50 Spectrum ATR Pike Technologies, in the range between $4000 \mathrm{~cm}^{-1}$ and $525 \mathrm{~cm}^{-1}$.

2.2.5. Thermal Analysis. Differential Scanning Calorimetry (DSC) analysis was performed on the Calorimeter Q100 (TA Instruments) from $0^{\circ} \mathrm{C}$ to $150^{\circ} \mathrm{C}$ at a heating rate of $10^{\circ} \mathrm{C} / \mathrm{min}$, under nitrogen atmosphere. Average of duplicates was used to determine the melting temperature and the enthalpy of fusion of each membrane. Crystallinity degree $(X c)$ of membranes was calculated by (1) [16]:

$$
X c(\%)=\frac{\Delta H_{m}}{\Delta H_{m}^{0}} * 100 \%,
$$


where $X c$ is crystallinity degree, $\Delta H_{m}$ is enthalpy of fusion of membrane, and $\Delta H^{0}{ }_{m}$ is enthalpy of fusion of pure crystalline chitosan.

2.2.6. Moisture Content. The percentage of moisture of the membranes was determined by the gravimetric method. The membranes were weighted before (initial mass) and after (final mass) being placed in an oven with air circulation at $105^{\circ} \mathrm{C}$ for 24 hours. The percentage of moisture was calculated by (2). The average of triplicates was used to calculate the moisture content of the samples:

$$
M(\%)=\left[\frac{\left(m_{i}-m_{f}\right)}{m_{i}}\right] * 100 \%,
$$

where $M(\%)$ is percentage of moisture, $m_{i}$ is initial mass, and $m_{f}$ is final mass.

2.2.7. Contact Angle. Contact angle measurements were performed to verify the affinity of the membranes with water. The test was carried out in a contact angle System OCADataPhysics Software SCA20 instrument using deionized water at $24^{\circ} \mathrm{C}$. Contact angle measurement was performed after a static time of 30 s. A tangent method and "Sessile Drop Fitting" in the software were applied to fit the drop shape and calculate the contact angle. For each membrane, three samples were used and six data points were taken for mean and standard deviation calculation.

2.2.8. Swelling Behavior. Samples were cut into pieces of $1 \mathrm{~cm}^{2}$ and placed in a desiccator with silica for $24 \mathrm{~h}$ and then weighed to determine dry mass. They were immersed in phosphate buffered solution (PBS) $\mathrm{pH} 7.2$ at $37^{\circ} \mathrm{C}$ for 24 and 72 hours in order to simulate physiological condition. The soaked samples were then blotted with filter paper to remove nonabsorbed surface water and then weighed again to determine the wet mass. The percentage of swollen was calculated by (3). The average of three replicates was used.

$$
S(\%)=\left[\frac{\left(m_{w}-m_{d}\right)}{m_{w}}\right] * 100 \%,
$$

where $S(\%)$ is percentage of swelling, $m_{w}$ is wet mass, and $m_{d}$ is dry mass.

2.2.9. Water-Vapor Permeability. The coefficient of permeability of water-vapor was determined gravimetrically according to the ASTM E96-95 [17]. Briefly, the thickness of the membranes was measured using a Digimatic Micrometer. Then, samples were cut to a size to cover the permeation cell orifice of $4.5 \mathrm{~cm}$ diameter, which resulted in a permeation area of $15.21 \mathrm{~cm}^{2}$, and fasten to the cells containing anhydrous calcium chloride. The cells were placed in a hermetically sealed container containing a supersaturated sodium chloride solution in order to maintain $75 \%$ of relative humidity at $25^{\circ} \mathrm{C}$. After two hours, cells were weighed and this procedure was repeated at regular intervals of time, for 72 hours. Watervapor permeability coefficient was calculated by (4) and average of triplicates was used.

$$
\mathrm{WVP}=\frac{(G \cdot \delta)}{\left(A \cdot \Delta \mathrm{P}_{w}\right)},
$$

where WVP is water-vapor permeability coefficient $\left(\mathrm{g} \cdot \mathrm{mm} / \mathrm{m}^{2} \cdot\right.$ day.kPa), $G$ is mass gain per day (g/day), $\delta$ is average thickness of membranes (mm), $A$ is exposed area to mass transfer $\left(\mathrm{m}^{2}\right)$, and $\Delta \mathrm{P}_{w}$ is partial pressure difference across the film $(\mathrm{kPa})$.

2.2.10. In Vitro Cytotoxicity. Indirect cytotoxicity was performed to evaluate the potential deleterious effects of substances released in the culture medium by the membranes. Thus, $10 \mathrm{mg}$ of each sample was separately placed in $10 \mathrm{~mL}$ of high glucose DMEM medium and incubated at $37^{\circ} \mathrm{C}$ in order to potentially release deleterious substances for 24 hours. HDF cells (ATCC ${ }^{\circledR}$ PCS-201- $010^{\mathrm{TM}}$ ), primary dermal fibroblasts derived from skin, were grown in high glucose DMEM medium (containing 10\% heat inactivated fetal bovine serum, L-glutamine, $\mathrm{NaHCO}_{3}$, pyridoxine- $\mathrm{HCl}$, $4.5 \mathrm{~g} / \mathrm{L}$ glucose, and $1 \%$ penicillin-streptomycin solution) as monolayer in a $75 \mathrm{~cm}^{2}$ cell culture flasks in the $37^{\circ} \mathrm{C}$ incubator under $\mathrm{CO}_{2}$. When up to $90 \%$ confluence, cells were harvested using $0.05 \%$ trypsin-EDTA, washed thrice with PBS (1200 rpm for 4 minutes), and resuspended in cell media. Then, cells were seeded in a 96-well plate at the density of $2 \times 10^{4}$ cells $/ 200 \mu \mathrm{L} /$ well using the same medium and incubated overnight at $37^{\circ} \mathrm{C}$ in the presence of $5 \% \mathrm{CO}_{2}$. After overnight growth, the medium was removed from each well and replaced with $180 \mu \mathrm{L}$ of fresh medium. Then, $20 \mu \mathrm{L}$ of the previously prepared sample was added (triplicates). For the positive control wells $200 \mu \mathrm{L}$ of fresh medium was added and $200 \mu \mathrm{L}$ of DI water was added to the negative control wells. The plate was placed back to the incubator and, after $24 \mathrm{~h}$, the medium was removed from all the wells and replaced with $200 \mu \mathrm{L}$ of fresh medium. Then, $20 \mu \mathrm{L}$ of Alamar blue reagent solution $(2 \mathrm{~mL}$ MTS $+100 \mu \mathrm{L}$ PMS $)$ was added to each of the wells. The plate was incubated for 3 hours and the fluorescence was read at $590 \mathrm{~nm}$ emission $(550 \mathrm{~nm}$ of excitation). Average of the triplicates was taken to calculate cells viability.

\section{Results and Discussion}

3.1. Extract of Unripe Banana Peels. The extraction of watersoluble compounds present in the unripe banana peel was performed by the decoction method, which consisted of boiling the peel in distilled water. After filtering to remove the extracted peels, a brownish solution was obtained as first extract. The browning of the solution can be attributed to the enzymatic oxidation of the tannins, which are present in the peels [18]. The extraction procedure was repeated three times and a decrease of the brownish color of the extracts was observed when compared to the first extract. Both first and third extracts were used to prepare the membranes. 


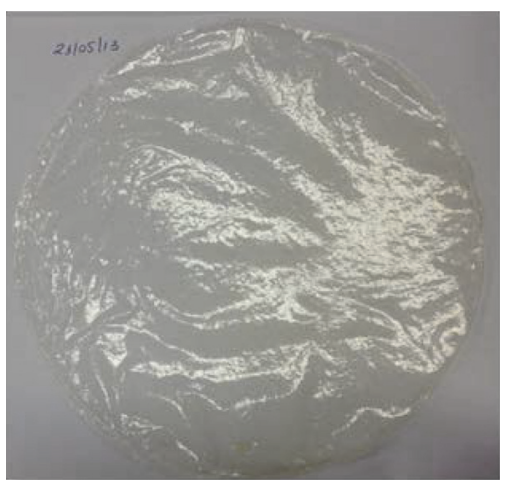

(a)

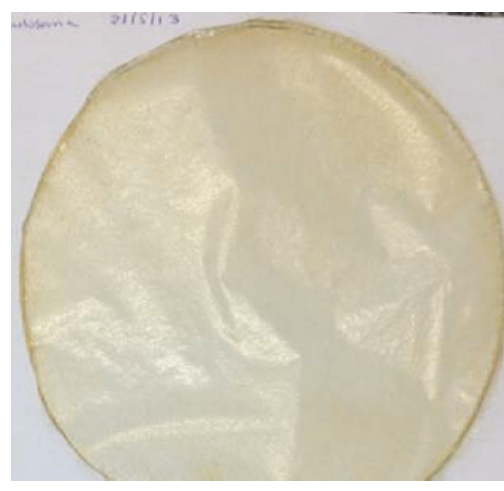

(b)

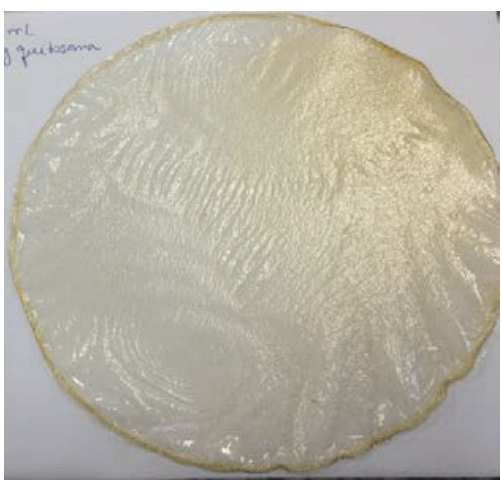

(c)

FIGURE 1: Chitosan membranes associated with the extract of unripe banana peel: (a) Chitosan membrane, CB0; (b) chitosan membrane prepared with the first extract, CB1; and (c) chitosan membrane prepared with the third extract, CB3.

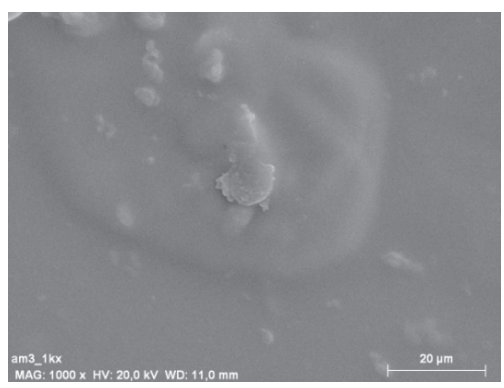

(a)

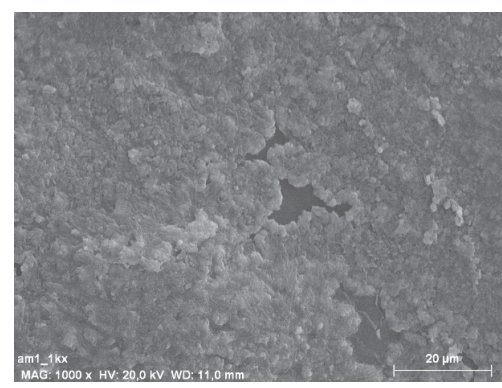

(b)

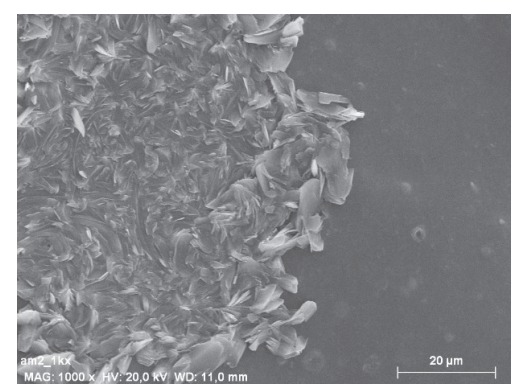

(c)

FIGURE 2: Scanning electron microscopy images of the membranes: (a) chitosan membrane, CB0; (b) chitosan membrane prepared with the first extract, CB1; and (c) chitosan membrane prepared with the third extract, CB3.

3.2. Preparation of the Membranes. The membranes were prepared by the solvent evaporation technique, by casting a solution in a circular mold. After drying, samples were easily unmolded. Flexible and uniform membranes were obtained and are shown in Figure 1. As expected, CB0 was transparent, while CB1 and CB3 were translucent due to the clear brown color. Kamel et al. (2016) also reported the brownish color of chitosan/banana peel powder nanocomposites after the filler addition [11]. Moreover, these authors reported an increase of the roughness of the nanocomposites due to the presence of the banana peel powder [11]. This increase of roughness was not observed for CB1 and CB3 membranes because of the different extraction method (decoction) used to obtain the extract of banana peel.

3.3. Morphological Analysis. SEM images of the membranes are shown in Figure 2. It can be observed that chitosan membrane (CB0) is dense, continuous, and homogeneous, while discontinuities and precipitates are found in CB1 and CB3. This may be attributed to the presence of the compounds extracted from the banana peel that structurally modified the chitosan matrix.

3.4. FTIR Analysis. Infrared Spectroscopy analysis was carried out to identify the functional groups found in the compounds extracted from the unripe banana peel. Figure 3 shows the spectra obtained for CB1, CB3, and unripe banana peel (powder). The spectra were collected in the range $4000-500 \mathrm{~cm}^{-1}$. A broad band observed in all spectra from 3500 to $3000 \mathrm{~cm}^{-1}$ can be attributed to the sum of the $-\mathrm{OH}$ stretching, which is characteristic of polyphenolic extracts [19]. In addition, $\mathrm{CH}, \mathrm{CH}_{2}$, and $\mathrm{CH}_{3}$ stretching vibrations derived from carbohydrates can be observed in the region of $2960-2925 \mathrm{~cm}^{-1}$ [20]. Nevertheless, these regions do not show conclusive evidences of the presence of tannins in unripe banana peel and membranes. The presence of phenols/tannins in CB1, CB3, and unripe banana peel spectra can be suggested by the presence of bands at $1600 \mathrm{~cm}^{-1}$ as characteristic bands of the aromatic skeleton chain, which can be attributed to the $\mathrm{C}=\mathrm{C}$ bond of the ring. The stretching of the $\mathrm{C}=\mathrm{C}-\mathrm{C}$ aromatic bond appears in the region of $1618-1449 \mathrm{~cm}^{-1}$ [19-21]. Kame et al. (2016) also reported a band at $1630 \mathrm{~cm}^{-1}$ in the banana powder spectrum [11]. The bands around $770 \mathrm{~cm}^{-1}$ found in $\mathrm{CB1}$ and banana peel spectra can also indicate the presence of aromatic rings. Moreover, bands between 1320 and $1340 \mathrm{~cm}^{-1}$ can be attributed to angular deformations C-O-H of phenols, derived from the pirate ring of tannins [20]. The stretching in $1340 \mathrm{~cm}^{-1}$ is due to the $\mathrm{C}-\mathrm{O}$ of typical of flavonoid C-rings [21]. Although FTIR analysis was not conclusive, 
TABLE 1: Thermal properties of raw materials and membranes: melting temperature and enthalpy of fusion of chitosan, unripe banana peel powder, and membranes obtained by Differential Scanning Calorimetry. Crystallinity degree (Xc) of membranes calculated by (1).

\begin{tabular}{lcccrr}
\hline Sample/property & Chitosan & Banana peel & CB0 & CB1 & CB3 \\
\hline $\operatorname{Tm}\left({ }^{\circ} \mathrm{C}\right)$ & 85.08 & 77.74 & 82.21 & 82.56 & 82.27 \\
$\Delta H_{m}(\mathrm{~J} / \mathrm{g})$ & 473.0 & 344.7 & 493.6 & 383.7 & 490.8 \\
$X c(\%)$ & - & - & 52.18 & 40.56 & 51.88 \\
\hline
\end{tabular}

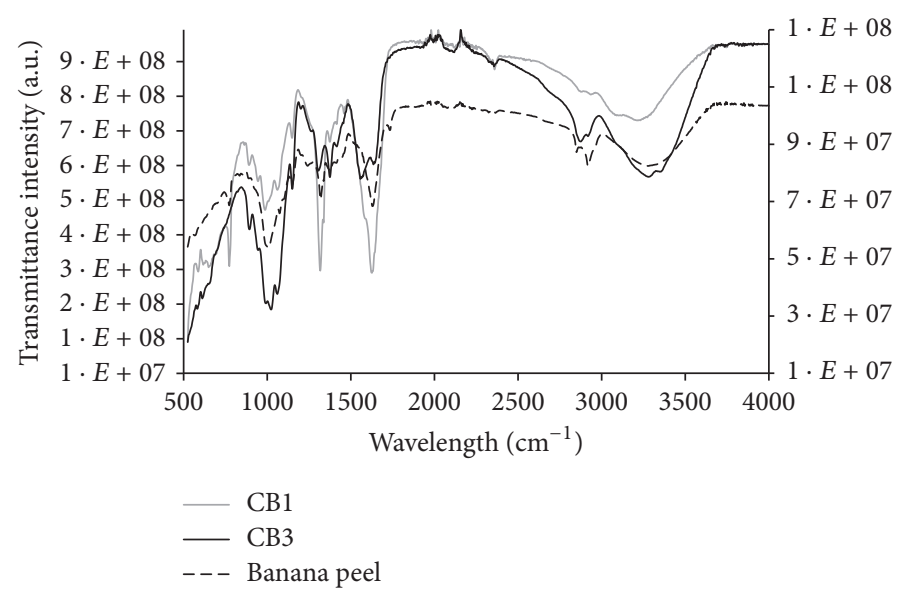

FIGURE 3: FTIR spectra of unripe banana peel and chitosan membranes prepared with the extracts of banana peel: (dashed) banana peel; (grey) chitosan membrane prepared with the first extract, CB1; and (black) chitosan membrane prepared with the third extract, CB3. Banana peel data is shown in the secondary transmittance intensity axis.

it showed strong evidences of the presence of tannins in unripe banana peel and membranes. Tannins show antimicrobial activity, which plays an important role in wound healing applications [9-13]. We recently demonstrated the efficiency of chitosan membranes associated with unripe banana peel extracts on the growth inhibition of Staphylococcus aureus and Escherichia coli, most common bacteria found in skin wounds [9]. Kamel et al. (2016) also reported the antimicrobial susceptibility of Escherichia coli, Staphylococcus aureus, Pseudomonas aeruginosa, and Candida albicans against chitosan/banana peel powder nanocomposites [11]. Kapadia et al. (2015) investigated the antimicrobial activity of banana peel on Porphyromonas gingivalis and Aggregatibacter actinomycetemcomitans in vitro [14]. They attributed the reported antimicrobial activity to the presence of bioactive compounds, such as flavonoids and tannins, found in banana peel $[14,22]$.

3.5. Thermal Analysis. Differential scanning calorimetry (DSC) results are shown in Table 1. The melting points (Tm) found for the membranes were well above physiological temperature $\left(37^{\circ} \mathrm{C}\right)$, suggesting good thermal stability. Moreover, similar melting temperatures were observed for $\mathrm{CB} 0, \mathrm{CB} 1$, and $\mathrm{CB} 3$, suggesting that the extracted compounds did not influence melting temperature. On the other hand, the fusion enthalpy of CB1 (383.7 J/g) was considerably lower than that found for $\mathrm{CB} 0$ and $\mathrm{CB} 3$ (493.6 J/g and $490.8 \mathrm{~J} / \mathrm{g}$, resp.). The reduced CB1 enthalpy of fusion indicates a lower crystallinity degree (Table 1 ) that can be explained by the presence of high concentration of extracted compounds (Figure 2). These compounds may act as plasticizers/impurities and make polymeric chains organization more difficult, reducing the crystallinity degree $[16,23]$. Crystallinity is an important parameter and may considerably affect mechanical and physical properties of the membranes.

3.6. Contact Angle. The experiments for contact angle measurements were performed in order to evaluate water affinity (hydrophilicity), an important parameter for wound dressings $[4,6,8]$. The contact angle indicates interfacial interaction between a solid and a liquid. Cohesive force of the liquid tends to form a spherical drop, while adhesive force of the solid tends to spread the water-drop [24]. Contact angle lower than $90^{\circ}$ indicates hydrophilic character of the solid whereas contact angle higher than $90^{\circ}$ is found for hydrophobic materials [24]. Time is also a parameter to be considered since the angle of contact may vary due to solidliquid interactions during the measurements [24]. Surface roughness also plays an important role in the contact angle [24].

Figure 4 shows contact angle measurements for CB0 at different times. As expected, the contact angle was decreasing with time, which can be attributed to water-membrane interaction and swelling. Table 2 shows the contact angle measurements for CB0, CB1, and CB3 at 30 s. All samples showed hydrophilic character (contact angle lower than $90^{\circ}$ ). Similar angles were measured for CB0 and CB3, while lower contact angle value was observed for CB1. This can be attributed to the presence of high concentration of extracted 
TABLE 2: Thickness, moisture content, contact angle, and water-vapor permeability coefficients of the membranes.

\begin{tabular}{lcccc}
\hline Sample & Thickness $(\mu \mathrm{m})$ & Percentage of moisture $(\%)$ & Contact angle $\left(^{\circ}\right)$ & WVP coefficient $\left(\mathrm{g} \cdot \mathrm{mm} / \mathrm{m}^{2} \cdot \mathrm{day} \cdot \mathrm{kPa}\right)$ \\
\hline CB0 & $88.47 \pm 6.85$ & $13.26 \pm 2.44$ & $68.21 \pm 1.42$ & $22.07 \pm 1.76$ \\
CB1 & $69.27 \pm 8.01$ & $13.84 \pm 2.07$ & $61.72 \pm 0.28$ & $14.08 \pm 1.04$ \\
CB3 & $58.06 \pm 6.20$ & $12.31 \pm 3.93$ & $66.40 \pm 1.45$ & $15.75 \pm 0.50$ \\
\hline
\end{tabular}
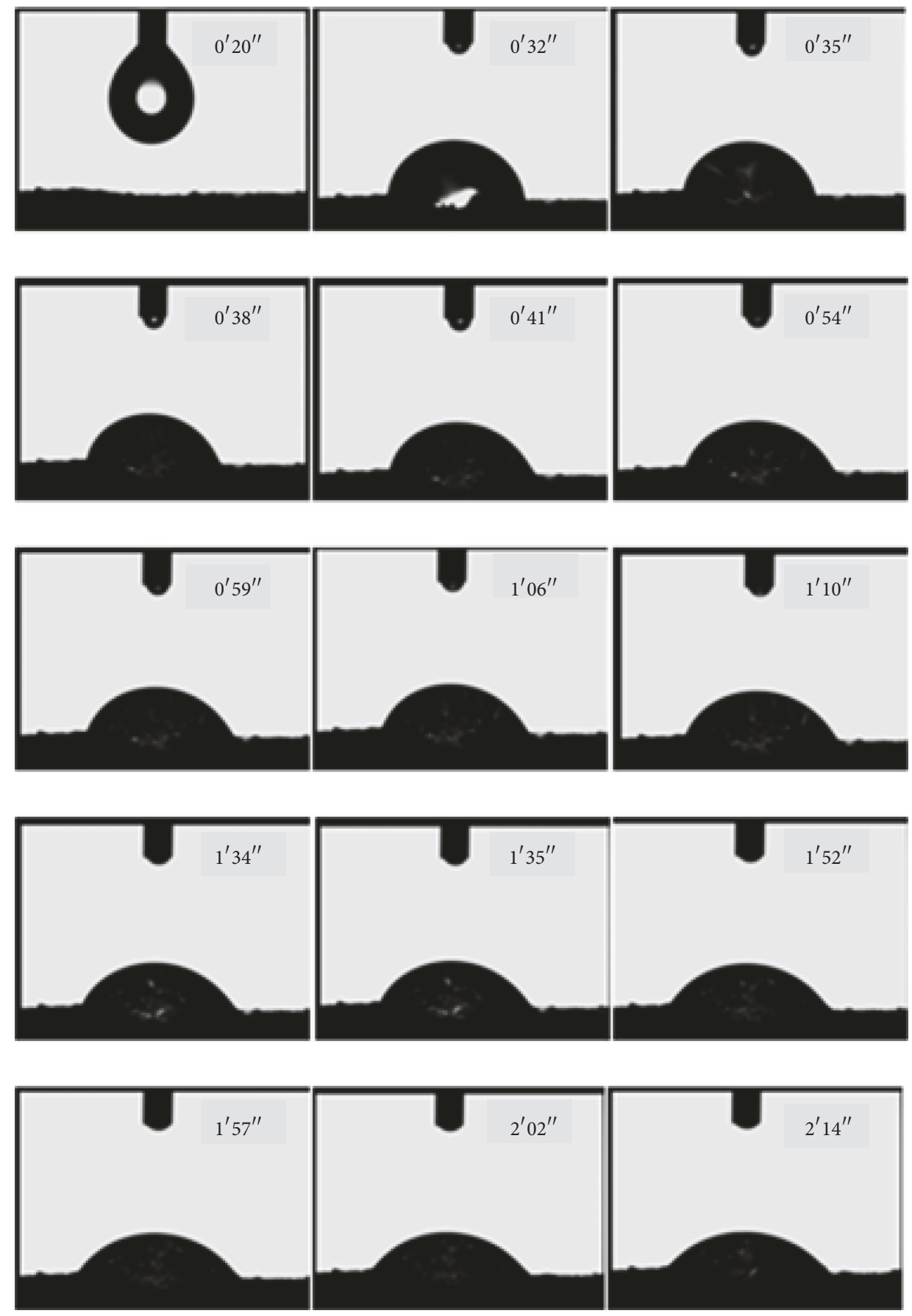

FIGURE 4: Contact angle images took at different times for the chitosan membrane, CB0. Total time: 2 minutes and 20 seconds. 


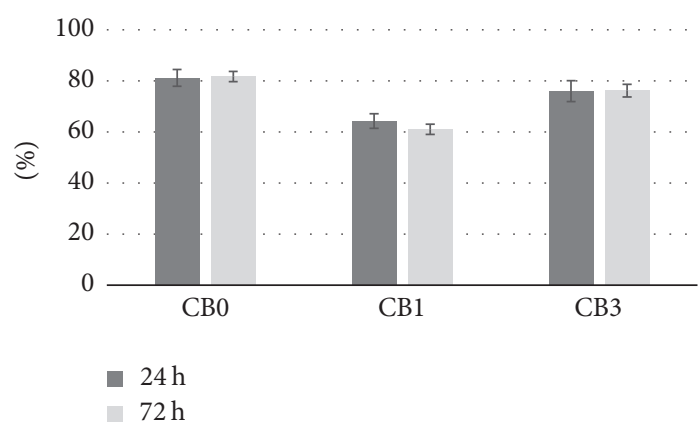

Figure 5: Swelling ratios of membranes after 24 and 72 hours obtained at simulated physiological conditions $\left(37^{\circ} \mathrm{C}, \mathrm{pH}\right.$ 7.2).

compounds that modified the roughness of this membrane as it can be seen in Figure 2.

Contact angle and roughness play an important role on cell adhesion since cells attachment in hydrophilic and rough surfaces is favorable. Thus, all membranes demonstrate adequate properties for potential cellular attachment in the wound site.

3.7. Moisture Content. Oven drying at standardized temperature $\left(105^{\circ} \mathrm{C}\right)$ is one of the methods to determine water content of a sample. The percentages of moisture of CB0, $\mathrm{CB} 1$, and CB3 are shown in Table 2. No significant difference was observed in the moisture content of the membranes, suggesting that the presence of extracted compounds did not influence their water content.

3.8. Swelling Behavior. On the other hand, the presence of the extract of unripe banana peel affected the water uptake of the membranes. It is important to mention that an ideal wound dressing should be able to keep the wound moist and absorb fluids and exudates $[4,6,8]$. The swelling ratios were calculated after 24 and 72 hours at physiological condition and the results are shown in Figure 5. All samples reached the swelling equilibrium at up to 24 hours since no significant changes in the swelling ratios were observed at 72 hours. Nevertheless, for both periods studied, considerable differences on swelling were observed. The water uptake was inversely proportional to the extract concentration. Thus, CB1 was swollen less due to the high concentration of extracted compounds. Similar behavior was observed for enthalpy of fusion, suggesting that these compounds interacted with the polymeric matrix, reducing crystallinity and water uptake due to chitosan-OH interactions. Kamel et al. (2016) also reported a decrease in the swelling of chitosan/banana peel powder nanocomposites. They suggested that some sort of interaction between negatively charged compounds in the banana peel powder and positively charged groups on the chitosan backbone may restrict the mobility of the chitosan chains, which decrease the water uptake [11].

3.9. Water-Vapor Permeability (WVP). In order to investigate the effects of the extract of unripe banana peels on the barrier properties of chitosan membranes, water-vapor permeability

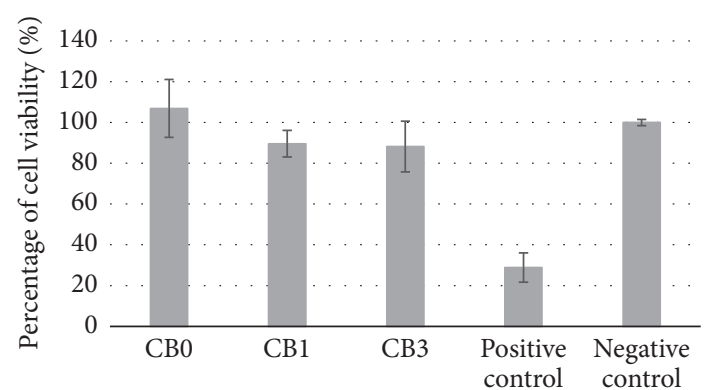

FIGURE 6: Indirect cytotoxicity of membranes against primary dermal fibroblasts.

experiment was carried out for 3 days according to the ASTM E95-96 [17]. WVP coefficients of CB0, CB1, and CB3 are shown in Table 2. The presence of extracted compounds on $\mathrm{CB} 1$ and $\mathrm{CB} 3$ reduced the water-vapor permeability coefficients of these membranes when compared to CB0. This may be due to the structural changes on the polymeric matrix caused by the presence of the extract (Figure 2). In addition, WVP of hydrophilic films may also be affected by swelling, which can reduce the resistance to mass transfer as observed for CB0. Kamel et al. (2016) also reported an increase in water resistance of chitosan/banana peel powder nanocomposites, which may reduce their WVP [11]. WVP rate of normal skin is around $0.85 \mathrm{mg} / \mathrm{cm}^{2} \cdot \mathrm{h}$ and ranges from 1.16 to $21.41 \mathrm{mg} / \mathrm{cm}^{2} \cdot \mathrm{h}$ for an injured skin [25]. One of the most important functions of a wound dressing is to avoid excessive evaporative water loss that may cause wound bed dehydration $[6,8]$. Moreover, the wound must be kept wet in order to accelerate healing [4]. Therefore, a wound dressing should have a WVP rate in the range of 8.33 to $10.42 \mathrm{mg} / \mathrm{cm}^{2} \cdot \mathrm{h}$ [25]. Thus, considering the partial pressure difference through the membranes and the thickness, CB0, CB1, and CB3 WVP rates are adequate for wound healing applications.

3.10. In Vitro Cytotoxicity. Indirect cytotoxicity test evaluates the potential deleterious effects of substances released in the culture medium by the membranes. Chitosan has been shown as biocompatible and nontoxic $[4,8,9]$. However, cytotoxicity evaluation of its association with unripe banana peel was not reported yet. Indirect cytotoxicity results (Figure 6) indicate that $\mathrm{CB} 0, \mathrm{CB} 1$, and $\mathrm{CB} 3$ did not release toxic substances to the culture media. In addition, Chitosan membrane (CB0) showed viable cell number higher than negative control of cytotoxicity, what can be attributed to chitosan's biological property of stimulating cell proliferation $[4,8,9]$. CB1 and CB3 showed over $80 \%$ of cell viability suggesting these membranes are nontoxic for dermal fibroblasts and may lead to potential biomedical applications in the area of skin wound dressing.

\section{Conclusion}

The preparation of chitosan membranes associated with the extracts of unripe banana peel was successfully demonstrated. The presence of tannins in the membranes was 
confirmed by the color change, SEM, and FTIR analyses. The membranes showed good thermal stability at physiological temperature and the $\mathrm{CB} 1$ degree of crystallinity was reduced by the presence of the extracted compounds. Contact angle measurements $\left(90^{\circ}\right)$ indicated hydrophilic character of the membranes, which permits cellular attachment in the wound site. All membranes were able to absorb fluids at physiological conditions and permit gaseous exchange, demonstrating WVP rates in the desired range for skin substitutes. In conclusion, indirect cytotoxicity against dermal fibroblasts was not observed for the membranes associated with the unripe banana peel extracts, suggesting their potential use as skin wound dressing.

\section{Conflicts of Interest}

The authors declare that they have no conflicts of interest.

\section{Acknowledgments}

The authors thank CNPq, CAPES, FAPEMIG, and UnifalMG for financial support. They also thank the Nanoscience Technology Center at UCF for the cell culture facility.

\section{References}

[1] L. M. Morton and T. J. Phillips, "Wound healing and treating wounds Differential diagnosis and evaluation of chronic wounds," Journal of the American Academy of Dermatology, vol. 74, no. 4, pp. 589-605, 2016.

[2] C. K. Sen, G. M. Gordillo, S. Roy et al., "Human skin wounds: a major and snowballing threat to public health and the economy," Wound Repair and Regeneration, vol. 17, no. 6, pp. 763-771, 2009.

[3] M. Frieri, K. Kumar, and A. Boutin, "Wounds, burns, trauma, and injury," Wound Medicine, vol. 13, pp. 12-17, 2016.

[4] M. B. Dreifke, A. A. Jayasuriya, and A. C. Jayasuriya, "Current wound healing procedures and potential care," Materials Science and Engineering $C$, vol. 48, pp. 651-662, 2015.

[5] D. Harper, A. Young, and C.-E. McNaught, "The physiology of wound healing," Surgery (United Kingdom), vol. 32, no. 9, pp. 445-450, 2014.

[6] H. C. Korting, C. Schollmann, and R. J. White, "Management of minor acute cutaneous wounds: importance of wound healing in a moist environment," Journal of the European Academy of Dermatology and Venereology, vol. 25, no. 2, pp. 130-137, 2011.

[7] D. J. Margolis, O. Hoffstad, J. Nafash et al., "Location, location, location: Geographic clustering of lower-extremity amputation among medicare beneficiaries with diabetes," Diabetes Care, vol. 34, no. 11, pp. 2363-2367, 2011.

[8] M. G. N. Campos, L. H. I. Mei, and A. R. Santos Jr, "Sorbitolplasticized and neutralized chitosan membranes as skin substitutes," Materials Research, vol. 18, no. 4, pp. 781-790, 2015.

[9] P. B. Franco, L. A. de Almeida, R. F. C. Marques, G. Brucha, and M. G. N. Campos, "Evaluation of antibacterial activity of chitosan membranes associated to unripe banana peel," Materials Science Forum, vol. 869, pp. 859-863, 2016.

[10] S. Someya, Y. Yoshiki, and K. Okubo, "Antioxidant compounds from bananas (Musa Cavendish)," Food Chemistry, vol. 79, no. 3, pp. 351-354, 2002.
[11] N. A. Kamel, S. L. A. El-messieh, and N. M. Saleh, "Chitosan/Banana peel powder nanocomposites for wound dressing application: preparation and characterization," Materials Science and Engineering: C, vol. 72, pp. 543-550, 2016.

[12] P. B. Lino, C. F. Correa, M. E. D. L. Archondo, and D. C. E. L. Dellova, "Evaluation of post-surgical healing in rats using a topical preparation based on extract of musa sapientum epicarp," Brazilian Journal of Pharmacognosy, vol. 21, no. 3, pp. 491-496, 2011.

[13] H. M. Maina, E. S. Heidi, and M. H. Shagal, "Analytical screening of nutritional and non-essential components in unripe and ripe fruits of banana (Musa sapientum)," International Journal of Medicinal Plant Research, vol. 1, no. 3, pp. 20-25, 2012.

[14] S. P. Kapadia, P. S. Pudakalkatti, and S. Shivanaikar, "Detection of antimicrobial activity of banana peel (Musa paradisiaca L.) on Porphyromonas gingivalis and Aggregatibacter actinomycetemcomitans: An in vitro study," Contemporary Clinical Dentistry, vol. 6, no. 4, pp. 496-499, 2015.

[15] H. W. Von Loesecke, Bananas, Interscience, NY, USA, 2nd edition, 1950.

[16] S. Ahmed, M. A. Sheraz, and I. U. Rehman, "Studies on tolfenamic acid-chitosan intermolecular interactions: effect of $\mathrm{pH}$, polymer concentration and molecular weight," AAPS PharmSciTech, vol. 14, no. 2, pp. 870-879, 2013.

[17] M. K. Kumaran, "Interlaboratory comparison of the astm standard test methods for water vapor transmission of materials (E96-95)," Journal of Testing and Evaluation, vol. 26, no. 2, pp. 83-88, 1998.

[18] V. Battestini, L. K. Matsuda, and G. A. Macedo, "Fontes e aplicações de taninos e tanases em alimentos," Alimentos e Nutrição, vol. 15, no. 1, pp. 63-72, 2004.

[19] K. Fernández and E. Agosin, "Quantitative analysis of red wine tannins using Fourier-transform mid-infrared spectrometry," Journal of Agricultural and Food Chemistry, vol. 55, no. 18, pp. 7294-7300, 2007.

[20] A. Ricci, K. J. Olejar, G. P. Parpinello, P. A. Kilmartin, and A. Versari, "Application of Fourier transform infrared (FTIR) spectroscopy in the characterization of tannins," Applied Spectroscopy Reviews, vol. 50, no. 5, pp. 407-442, 2015.

[21] L. Falcão and M. E. M. Araújo, “Tannins characterization in historic leathers by complementary analytical techniques ATRFTIR, UV-Vis and chemical tests," Journal of Cultural Heritage, vol. 14, no. 6, pp. 499-508, 2013.

[22] A. Pereira and M. Maraschin, "Banana (Musa spp) from peel to pulp: ethnopharmacology, source of bioactive compounds and its relevance for human health," Journal of Ethnopharmacology, vol. 160, pp. 149-163, 2015.

[23] Y. Yuan, B. M. Chesnutt, W. O. Haggard, and J. D. Bumgardner, "Deacetylation of chitosan: material characterization and in vitro evaluation via albumin adsorption and pre-osteoblastic cell cultures," Materials, vol. 4, no. 8, pp. 1399-1416, 2011.

[24] Y. Yuan and T. R. Lee, "Contact angle and wetting properties," in Surface Science Techniques, G. Bracco and B. Holst, Eds., vol. 51 of Springer Series in Surface Sciences, pp. 3-34, Springer, Berlin, Germany, XXIII, 2013.

[25] B. Singh and L. Pal, "Sterculia crosslinked PVA and PVApoly(AAm) hydrogel wound dressings for slow drug delivery: mechanical, mucoadhesive, biocompatible and permeability properties," Journal of the Mechanical Behavior of Biomedical Materials, vol. 9, pp. 9-21, 2012. 

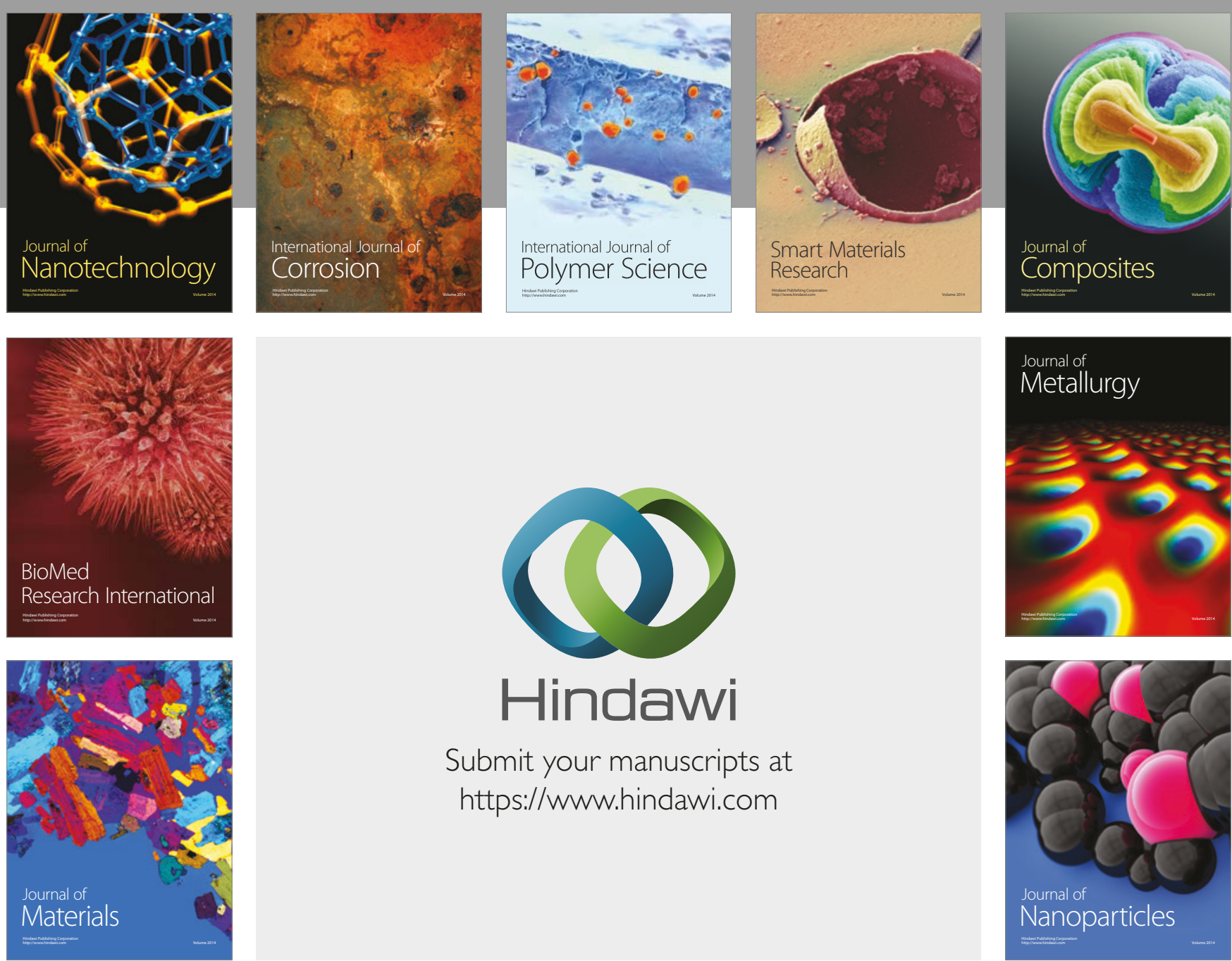

\section{Hindawi}

Submit your manuscripts at

https://www.hindawi.com
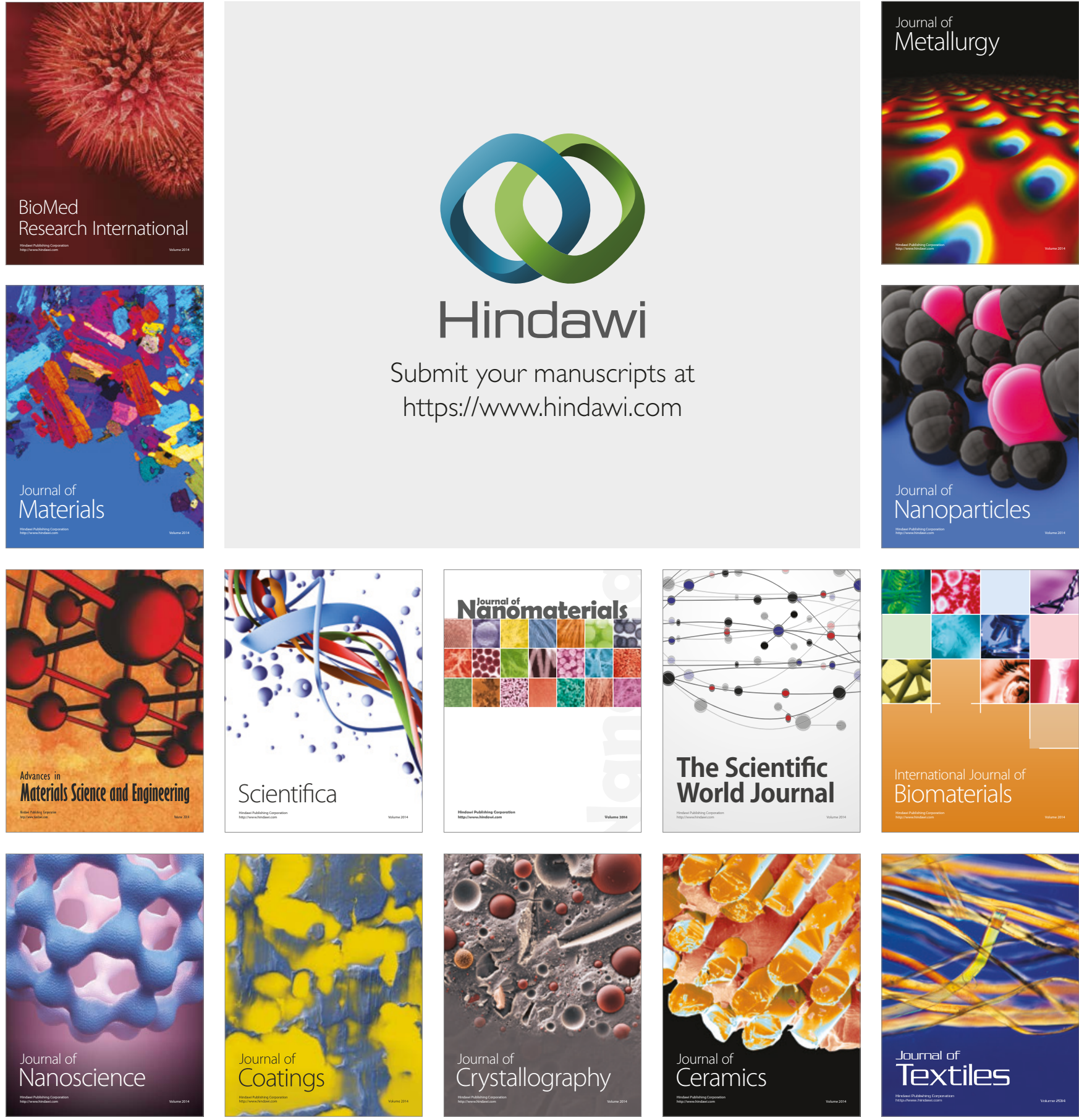

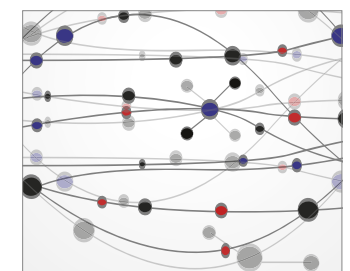

The Scientific World Journal
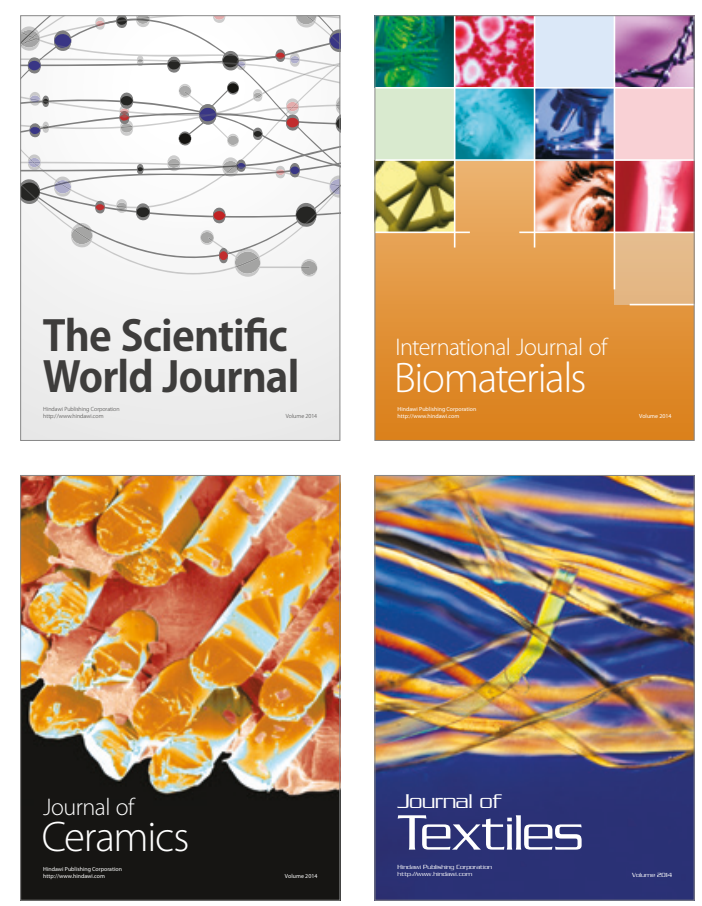